\title{
Analysis of Link-Layer Backoff Algorithms on Point-to-Point Markov Fading Links: Effect of Round-Trip Delays
}

\author{
A. Chockalingam* and M. Zorzi ${ }^{\dagger}$ \\ * Department of ECE, Indian Institute of Science, Bangalore 560012, India \\ ${ }^{\dagger}$ DEI, Università di Padova, via Gradenigo, 6/a - 35131 Padova, Italy
}

\begin{abstract}
Backoff algorithms can be employed on point-to-point wireless fading links to improve energy efficiency, particularly when the link experiences long deep fades and bursty errors. A backoff scheme at the link layer (LL), applying an appropriate backoff rule upon each LL packet loss event due to channel errors, can intentionally leave the channel idle (i.e., not transmit) for some specified number of slots, thereby reducing the possible energy wastage due to packet transmissions in error. Our new contribution in this paper is that we consider the use of backoff algorithms on wireless fading links with large round-trip delays, propose a Go-back-N (GBN) protocol with backoff, and present a renewal-reward analysis of the throughput and energy efficiency performance of the proposed scheme. We show that the GBN protocol with a linear backoff (LBO) strategy results in energy savings of about $2 \mathrm{~dB}$ compared to GBN with no backoff (NBO), even in the case of large round-trip delays. In addition, we also propose and analyze an adaptive Go-back-N/Stopand-Wait (GBN/SAW) ARQ scheme with LL backoff. We show that the proposed adaptive GBN/SAW ARQ scheme with LBO achieves energy efficiency performance quite close to that of an ideal (though not practical) backoff scheme which assumes a priori knowledge of the channel status in each slot.
\end{abstract}

Keywords - Backoff, energy efficiency, ARQ, Go-back-N, Stop-and-Wait, Markov fading, round-trip delay.

\section{INTRODUCTION}

Backoff algorithms can be employed beneficially on pointto-point wireless fading links. The motivation for backoff on point-to-point wireless links arises from the potential for energy savings when the link experiences long deep fades [1] and bursty errors. A backoff algorithm at the link layer (LL), applying an appropriate backoff rule upon each LL packet error event due to channel errors, can intentionally leave the channel idle (i.e., not transmit) for some specified number of slots, thereby reducing the possible energy wastage due to packet transmissions in error. In [2], we analyzed the throughput and energy efficiency performance of three LL backoff algorithms, namely, linear backoff (LBO), binary exponential backoff (BEBO) and geometric backoff (GBO), on point-topoint wireless fading links, assuming instantaneous feedback (i.e., zero round-trip delay). We computed the throughput and energy efficiency as the reward rates in a renewal process, and showed that the backoff algorithms can result in substantial energy savings. This analysis was extended to the UDP layer in [3], where energy efficiency was shown to be achieved even at the transport layer while using backoff algorithms at the link layer. The analyses in [2],[3] assume zero round-trip delay (RTD). Although propagation delays can be small in cellular/WLAN environments, processing delays in network elements can cause large RTDs. Hence, our main focus in this paper is to consider the use of LL backoff algorithms along with LL error control for the case of large round-trip delays. Specifically, we consider Go-back-N (GBN) ARQ with LL backoff. GBN ARQ has the advantage of being simpler to implement and analyze compared to selective repeat (SR) ARQ, yet GBN gives performance close to that of SR ARQ.

Our new contribution in this paper is that we consider the use of LL backoff algorithms for large RTDs, propose a Go-back$\mathrm{N}$ (GBN) protocol with LL backoff, and present a renewalreward analysis of the throughput and energy efficiency performance of the proposed scheme. We show that the GBN protocol with a linear backoff (LBO) strategy results in energy savings of about $2 \mathrm{~dB}$ compared to GBN with no backoff (NBO), even in the case of large RTDs. In addition, we also propose and analyze an adaptive Go-back-N/Stop-and-Wait (GBN/SAW) ARQ scheme with LL backoff. We show that the proposed adaptive GBN/SAW ARQ scheme with LBO achieves energy efficiency performance quite close to that of an ideal (though not practical) backoff scheme which assumes a priori knowledge of the channel status in each slot.

\section{LL BACKOFF ALGORITHMS}

We are interested in LL ARQ designs that exploit channel memory with a motivation to improve energy efficiency. The basic idea proposed is to remain idle (not transmit) if the channel condition is poor. The channel status is derived through ACK/NACK in LL ARQ. The transmitter idles incrementally more if the channel remains poor for a long time (e.g., as in the case of high channel memory for a shadowed pedestrian user). Backoff algorithms can be employed in such scenarios to determine the amount of time to idle. For example, the amount of backoff (or idle) time can be increased in direct proportion to the number of retransmission attempts of a $\mathrm{LL}$ packet in error. We consider that the LL packets are of constant size and that the time axis is split into slots of duration equal to one LL packet. Accordingly, the backoff time is expressed in terms of number of slots. Backoff time can be deterministic (e.g., linear backoff, binary exponential backoff) or probabilistic (e.g, geometric backoff), as described below.

Linear Backoff (LBO): In a linear backoff scheme, on the $i$ th successive failure of a LL packet, the LL leaves the channel idle for $i$ subsequent slots, i.e., the backoff delay grows linearly upon each successive failure.

Binary Exponential Backoff (BEBO): In this scheme, the LL leaves the channel idle for $2^{i}-1$ slots upon the $i$ th successive failure of a LL packet. 
Geometric Backoff (GBO): In this scheme, there is a parameter $g, 0 \leq g<1$. Following an idle or a LL packet failure, the LL leaves the channel idle in the next slot with probability $g$ (or equivalently, transmits a packet with probability $1-g$ ).

The tradeoff involved in these backoff algorithms is that we may loose some LL throughput (because of the possibility of remaining idle in good slots), but will save energy (because of not transmitting during bad slots). The throughput loss, however, is typically small, while energy savings may be significant, particularly when the channel fades are highly correlated and the round-trip delay is negligible [2]. In the following sections, we present the analysis of LBO for the case of large round-trip delay. Analysis of the other backoff strategies (BEBO, GBO) can be carried out likewise.

\section{BACKOFF STRATEGY}

In this section, we present the backoff strategy for the case of large round-trip delays. We assume that the ACK/NACK feedback for each LL packet sent is received at the sender $m$ slots after the packet transmission was initiated. In other words, the round-trip propagation and processing delay (which may include additional transmission delays, e.g., as in the wireline part of a $3 \mathrm{G}$ radio access network) is $m$ slots. Note that $m=1$ corresponds to the case of instantaneous feedback assumption [2]. We consider Go-back-N ARQ with window size equal to $m$, the number of round-trip and processing slots, $m>1$. The backoff strategy adopted in the case of $m>1$ (i.e., large RTD) is explained as follows.

It is noted that it is not obvious how to include backoff in GBN ARQ for the case of $m>1$ (it is quite straightforward in the zero RTD case, i.e., $m=1$ ). One way is to apply backoff to all failed packets (including those packets which failed during transmission in the window of $m$ RTD slots). An alternative, simpler way is to apply backoff only to the 1st failed packet in a transmission window. We select the latter strategy (i.e., apply backoff only to the 1st failed packet in the transmission window) based on the fact that all we should do is to leave empty slots as soon as we detect an error. In Sec. IV, we analyze this backoff strategy, and, in Sec. V, we present performance results to show that this strategy gives good energy savings. As an example, the operation of the GBN protocol with linear backoff is as follows (other backoff schemes work similarly).

As per GBN, after the first packet is sent in the first slot, $m-1$ more packets are also sent in the subsequent $m-1$ slots. The ACK/NACK for the first packet arrives at the beginning of the $(m+1)$ th slot. One of the following things can happen:

1) All the $m$ packet transmissions are successful, in which case the protocol continues as before.

2) The first packet fails. All the packets from the first packet onwards have to be retransmitted (regardless of the success or failure of the packets sent during the $m-1$ RTD slots). In this case, backoff is applied to the first packet, i.e.,

- $(m+1)$ th slot is left idle;

- first packet is retransmitted in slot $(m+2)$;
- 2nd to $m$ th packets are resent in the subsequent $m-1$ slots;

- if the first packet fails again in the $(m+2)$ th slot (which will be known through the NACK at the beginning of $(2 m+3)$ th slot $)$, then slots $(2 m+3)$ and $(2 m+4)$ are left idle (linear growth in backoff), following which the first packet is retransmitted again followed by transmissions of 2 nd to $m$ th packet in the subsequent slots, and so on.

3) The first packet succeeds, and one or more packets fail during the $m-1$ RTD slots. Say, the FIRST FAILURE in this $(m-1)$-slot window occurs in the $i$ th slot, $1<$ $i \leq m$. This failure will be known through the NACK received at the beginning of the $(i+m)$ th slot. Now backoff will be applied to this FIRST FAILED packet as described in 2) above.

\section{Performance Analysis}

In this section, we analyze the throughput and energy efficiency performance of the LL backoff algorithms for the case of $m>1$ (i.e., large RTD). Consider bulk data to be transmitted over a point-to-point wireless link. LL packets are of same size. The time axis is split into slots of duration equal to one LL packet duration. ACK/NACK feedback for each LL packet sent is assumed to be received error-free at the sender $m$ slots after the packet was sent ${ }^{1}$.

Markov Block Fading Model [4]: We employ a first-order Markov chain representation of the wireless channel with Markov parameters $p$ and $(1-q)$ being the probabilities that the packet transmitted in the $k$ th slot is a success given the packet transmitted in the $(k-1)$ st slot is a success or a failure, respectively. In other words, the channel transition probability matrix is

$$
M_{c}=\left[\begin{array}{ll}
p_{00} & p_{01} \\
p_{10} & p_{11}
\end{array}\right],
$$

where $p_{i j}, j \in\{0,1\}$ denotes the transition probability of moving from state $i$ to state $j$, and ' 0 ' denotes success state and ' 1 ' denotes failure state. That is, $p_{00}=p, p_{01}=(1-p)$, $p_{10}=(1-q)$, and $p_{11}=q$.

\section{A. Renewal Analysis}

In GBN, not all slots are candidates for successful transmissions. More specifically, even though the channel can be good in a slot, this does not necessarily lead to a positive contribution to the throughput due to the protocol rules (e.g., the slots immediately after a channel failure are lost regardless of the channel conditions). Define a throughput opportunity as a slot which, if the channel is good, will positively contribute to the throughput count. ${ }^{2}$

According to the protocol description and due to the Markov channel model, the packet transmission process is a renewal

\footnotetext{
${ }^{1}$ It is noted that $m$ denotes the time from when a packet transmission starts to when the corresponding feedback is known. In particular, the instantaneous feedback case corresponds to $m=1$.

${ }^{2}$ As an example, starting from a good channel state, all slots until (and including) the first bad slot are throughput opportunities, whereas the $m-1$ slots following the first bad slot are not, since even in the presence of good channel conditions the corresponding transmissions are wasted.
} 
process where the renewal instants are the times at which a packet is transmitted for the first time in a throughput opportunity. As such, we can count one packet success at each renewal instant, and the throughput (number of successfully delivered packets per slot) is the inverse of the average number of slots between successive renewals. Here, we analytically derive the statistics of the inter-renewal times for the schemes considered in this paper.

1) Ideal Backoff (IBO): Consider an ideal backoff (IBO) scheme, where we assume that the sender has a priori knowledge of the channel status in each slot, and it does not transmit in bad slots. Such a priori knowledge is impractical. However, this scheme is considered because it results in the best energy savings possible due to backoff (since no transmissions occur in any bad slot), which can act as a benchmark to compare the energy savings achieved in practically implementable backoff algorithms like LBO.

In IBO, consider the case in which a slot contains a successful transmission, i.e., the slot is a successful channel slot and is a throughput opportunity. Assuming that the packet transmission in the current slot $(t=0)$ is successful, we need to determine what is the average time until the next successful packet transmission. After the current slot (which counts as one), if the following slot $t=1$ is a failure the time before the next retransmission of the failed packet can be computed as follows. There are $m$ transmission slots (the one with the failed packet and the $m-1$ subsequent slots) in all cases. The retransmission occurs in the first good channel slot after then. With probability $p_{10}(m)$, the first available slot $(t=m+1)$ is a good one, and the corresponding delay is $m+1$. With probability $p_{11}(m)$, slot $m+1$ is bad, and for the next good slot one needs to wait for a geometric number of slots, with average $1 / p_{10}$. This leads to the following expression for the average time between two successful transmissions:

$$
1+p_{01}\left(m+\frac{p_{11}(m)}{p_{10}}\right) \text {. }
$$

For the average number of packet transmissions, we reason as before, except that we do not count the geometric number of slots (during which no transmissions occur), so we have

$$
1+p_{01} m \text {. }
$$

2) Deterministic Backoff: In this case, following the $j$-th failure, the next transmission attempt is performed after $d_{j}$ idle slots. According to the definition of the backoff schemes, we have $d_{j}=j$ for LBO.

Consider the following set of states (see Fig. 1): state $G$ corresponds to a successful transmission of a packet (i.e., a packet which is counted as useful throughput according to the protocol rules), whereas states $B_{j}, j=1,2, \ldots$, correspond to the subsequent failed attempts of a packet. That is, as long as packets are correctly sent, the protocol stays in state $G$. When a packet transmission encounters a bad channel and fails, the protocol goes to state $B_{1}$. From state $B_{1}$, if the next attempt to transmit the packet $\left(m+d_{1}\right.$ slots later according to the BO mechanism) is successful, the protocol goes back to state $G$, otherwise it goes to state $B_{2}$, where it

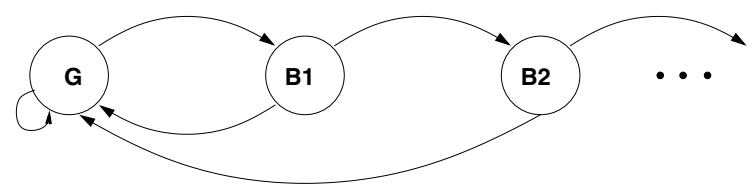

Fig. 1. Flow diagram for GBN with backoff

stays for $m+d_{2}$ slots, and so on. More specifically, the transition structure through this diagram is as follows. From state $G$ we can go back to state $G$ with probability $p_{00}$ or to state $B_{1}$ with probability $p_{01}$. From state $B_{j}$ we can go to state $G$ with probability $p_{10}\left(m+d_{j}\right)$ or to state $B_{j+1}$ with probability $p_{11}\left(m+d_{j}\right)$. The time associated with a visit to state $G$ is a single slot, whereas for state $B_{j}$ it is $m+d_{j}$ slots.

Suppose a packet transmission has failed and the protocol goes to state $B_{1}$. We want to compute the average time it takes until state $G$ is again reached. Note in fact that this is the first valid transmission to be counted in throughput, and in fact each visit to state $G$ is counted as a successful packet.

From state $B_{1}$, the system will visit a number of $B$ states until it eventually goes back to state $G$. The probability that the last $B$ state visited is $B_{j}$ (i.e., that the packet is correctly received at its $(j+1)$ st transmission, given that the first transmission was a failure) is found as

$$
\left(\prod_{i=1}^{j-1} p_{11}\left(m+d_{i}\right)\right) p_{10}\left(m+d_{j}\right)
$$

and the associated average time to go from $B_{1}$ to $G$ is then computed as

$$
D_{B G}=\sum_{j=1}^{\infty}\left(j m+\sum_{i=1}^{j} d_{i}\right)\left(\prod_{i=1}^{j-1} p_{11}\left(m+d_{i}\right)\right) p_{10}\left(m+d_{j}\right) .
$$

The corresponding number of packet transmissions is computed similarly, taking into account that in the backoff slots no transmissions occur:

$$
T_{B G}=\sum_{j=1}^{\infty} j m\left(\prod_{i=1}^{j-1} p_{11}\left(m+d_{i}\right)\right) p_{10}\left(m+d_{j}\right) .
$$

Finally, considering the visits to state $G$ as the renewal instants, we want to compute the average time between successive visits. This time is equal to one slot if the next transmission is successful, i.e., with probability $p_{00}$, whereas it is equal to $1+D_{B G}$ with probability $p_{01}$, i.e.,

$$
D_{G G}=1+p_{01} D_{B G}
$$

Similarly, for the number of transmissions between successive visits to state $G$ we have

$$
T_{G G}=1+p_{01} T_{B G} .
$$

Finally, the throughput and the energy efficiency of the scheme can be found as $1 / D_{G G}$ and $1 / T_{G G}$, respectively. The appropriate choice of the variables $d_{i}$ will correspond to the various backoff schemes, with the scheme without backoff (NBO) corresponding to $d_{i}=0$ for all $i$ (notice that in this case we have $T_{G G}=D_{G G}$ ). 


\section{B. Adaptive $S A W / G B N$ scheme}

This scheme is a modified version of the previous one. The idea is to use GBN (aggressive ARQ) in good channel conditions and SAW (non-aggressive ARQ) in bad channel conditions. GBN is used till a loss is detected. SAW is used till the lost packet is retransmitted successfully. Once successfully retransmitted, the protocol switches back to GBN, and so on.

This adaptive GBN/SAW scheme uses the same concept as in [5], adding backoff to it. As long as correct transmissions occur, the system stays in state $G$ as before. As soon as there is an erroneous transmission, the system goes to state $B_{1}$. The transition structure from there and the times associated to the various states are the same as before, except for two differences: 1) the number of packet transmissions per transition is now 1 instead of $m$ for all transitions from the $B$ states (SAW instead of GBN);2) after the eventual correct retransmission of a packet the GBN mode cannot be immediately restored, as the success of that transmission takes $m$ slots to be known at the transmitter. This second point requires that an additional state be introduced, as was done in [5]. This state, that we call $G^{\prime}$, corresponds to a correct transmission but is associated to a time of $m$ slots instead of only one. From state $G^{\prime}$, the system goes to state $G$ with probability $p_{00}(m)$, while it goes to state $B_{1}$ with probability $p_{01}(m)$.

The evolution of the system is therefore the following: when leaving state $G$ the system goes to state $B_{1}$, from where it eventually reaches state $G^{\prime}$. From there, the system either goes once again to $B_{1}$, or ends the cycle going to $G$. The number of times the system loops between $B_{1}$ and $G^{\prime}$ is a geometric random variable with mean $1 / p_{00}(m)$. The average time taken in going from $B_{1}$ to $G^{\prime}$ is

$$
D_{B G^{\prime}}=\sum_{j=1}^{\infty}\left(j m+\sum_{i=1}^{j} d_{i}\right)\left(\prod_{i=1}^{j-1} p_{11}\left(m+d_{i}\right)\right) p_{10}\left(m+d_{j}\right) \text {, (9) }
$$

and the average number of transmissions is

$$
T_{B G^{\prime}}=\sum_{j=1}^{\infty} j\left(\prod_{i=1}^{j-1} p_{11}\left(m+d_{i}\right)\right) p_{10}\left(m+d_{j}\right)
$$

where we took into account that the retransmissions are done using SAW, i.e., one per transition. The total delay between two consecutive visits to state $G$ is then found as

$$
D_{G G}=1+\frac{m+D_{B G^{\prime}}}{p_{00}(m)} p_{01} \text {. }
$$

where we accounted for the fact that the number of $B_{1}-G^{\prime}$ loops is a geometric r.v., and that each loop on average lasts $D_{B G^{\prime}}$ plus the $m$ slots for the transition from $G^{\prime}$ to $B_{1}$ (which in the last case leads to $G$ instead but has the same duration). A similar argument leads to

$$
T_{G G}=1+\frac{m+T_{B G^{\prime}}}{p_{00}(m)} p_{01} .
$$

Finally, note that in this case the successful transmissions correspond to visits to both states $G$ and $G^{\prime}$. Therefore, during the evolution between two consecutive visits to state $G$ we may have more than one success. The average number of successes is found as

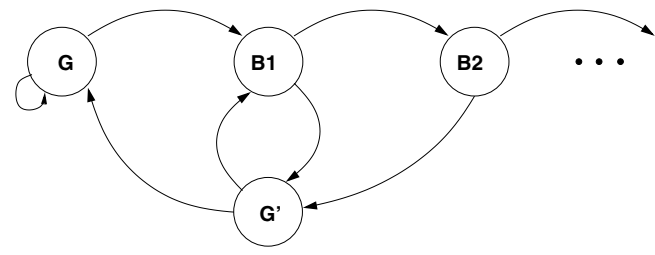

Fig. 2. Flow diagram for Adaptive GBN/SAW with backoff

$$
S_{G G}=1+\frac{1}{p_{00}(m)} p_{01}
$$

Therefore, the throughput and energy efficiency can be easily calculated as $S_{G G} / D_{G G}$ and $S_{G G} / T_{G G}$, respectively.

\section{RESUlts AND Discussion}

In this section, we present the analytical and simulation results of the throughput and energy efficiency performance. Fig. 3 shows the LL throughput performance of GBN without and with LL backoff, as a function of the number of RTD slots, $m$. The following three cases are considered: 1) GBN with Ideal Backoff (IBO), 2) GBN with No Backoff (NBO), and 3) GBN with Linear Backoff (LBO). We analytically computed the throughput and energy efficiency using channel parameters $p$ and $q$ corresponding to a fade margin of $3 \mathrm{~dB}$ and a normalized Doppler bandwidth $f_{d} T=0.001$ (which represents a high channel correlation scenario). For the first-order Markov representation of the multipath Rayleigh fading, the relation between average packet error rate $(\epsilon)$, fade margin $(F)$, and parameters $p$ and $q$ are given by [4]: $\epsilon=1-e^{-1 / F}=(1-p) /(2-p-q)$ and $(1-q)=$ $[Q(\theta, \rho \theta)-Q(\rho \theta, \theta)] /\left(e^{1 / F}-1\right)$, where $\theta=\sqrt{2 / F\left(1-\rho^{2}\right)}$, $\rho=J_{0}\left(2 \pi f_{d} \tau\right)$ is the correlation coefficient of two samples of the complex amplitude of the Rayleigh fading process taken $T$ seconds apart, $f_{d}$ is the Doppler bandwidth (ralated to user speed $v$ and carrier wavelength $\lambda$ as $\left.f_{d}=v / \lambda\right), J_{0}(\cdot)$ is the Bessel function of the first kind and zeroth order, and $Q(\cdot, \cdot)$ is the Marcum $Q$ function. At a carrier frequency of $900 \mathrm{MHz}, f_{d} T=0.001$ corresponds to user speed of 1.2 $\mathrm{Km} / \mathrm{h}$, link speed of $1 \mathrm{Mbps}$ and LL packet size of 1000 bits. Performance plots for the independent (i.i.d.) fading case are also computed and plotted. In Fig. 3, both analytical and simulation results agree well which validates the analysis.

From Fig. 3, the following observations can be made. As expected, GBN with IBO gives the best LL throughput performance both for $f_{d}=0.001$ as well as i.i.d. For $f_{d} T=0.001$, the performance of GBN with NBO is quite close to that of GBN with IBO. We pointed out earlier that LBO may lose some throughput (due to the possibility of remaining idle during good slots) compared to NBO. However, as can be seen in Fig. 3, this throughput loss for LBO compared to NBO turns out to be quite insignificant when the channel correlation is high $\left(f_{d} T=0.001\right)$, i.e., there is no major throughput loss due to backoff. On the other hand, the backoff results in significant energy savings as can be observed in Fig. 4.

For the same schemes and system parameters in Fig. 3, Fig. 4 shows the energy efficiency (normalized by the fading margin, $F$ ) as a function of the number of round-trip delay slots, $m$. It is noted that for $m=1$, the best possible energy efficiency is $1 / F$ (i.e., no transmissions in any of the bad slots) 


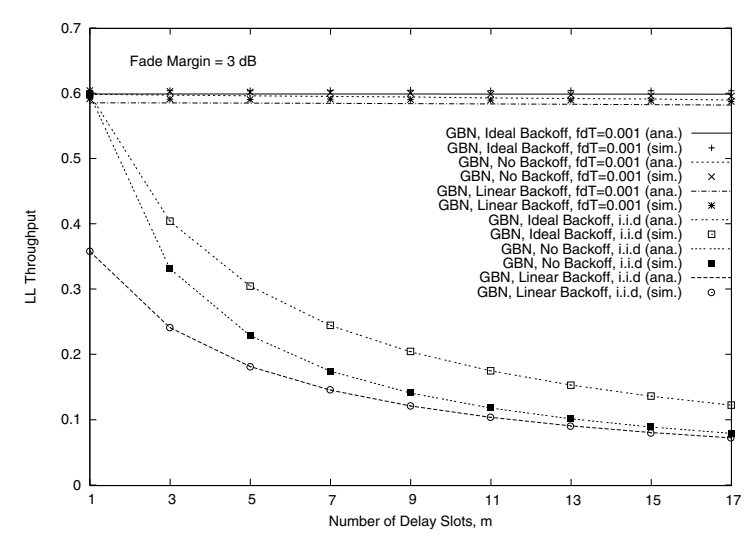

Fig. 3. LL Throughput as a function of number of round-trip delay slots, $m$, for GBN protocol without and with LL backoff.

which is achieved by IBO. The energy efficiency achieved is much less (equal to $(1-\epsilon) / F$ ) for $m=1$ when no backoff is performed (NBO). It can further be observed that when linear backoff is performed (LBO), the energy efficiency improves by about $2 \mathrm{~dB}$ compared to NBO. In fact, for $m=1$, LBO achieves energy efficiency close to that of IBO. But this energy efficiency improvement of LBO over GBO decreases as $m$ increases. This is because GBN is applied even during loss recovery - i.e., a retransmission attempt after a backoff involves transmission in $m$ slots, and when channel is highly correlated all these $m$ slot transmissions can be lost. This reduces the energy efficiency of LBO with increasing $m$. This performance behaviour motivates us to use a less aggressive ARQ (e.g., SAW) during loss recovery (bad periods) to get closer to the energy efficiency bound of IBO. Accordingly, adaptive GBN/SAW schemes without and with backoff are considered in Figs. 5 and 6.

Fig. 5 shows the energy efficiency of the adaptive GBN/SAW without and with backoff in comparison with GBN without and with backoff for $f_{d} T=0.001$. Fig. 6 shows the corresponding LL throughput. From Fig. 5 it can be observed that the energy efficiency of adaptive GBN/SAW with no backoff (i.e., no backoff during SAW phase) gets close to that of IBO for large $m$, but for small $m$, the energy efficiency is still poor (closer to NBO). This indicates that further improvement may be possible for small $m$ if backoff is introduced during SAW phase (i.e., adaptive GBN/SAW with backoff). As can be seen, the adaptive GBN/SAW with LBO almost achieves the energy efficiency bound of IBO at all values of $m$. This improved energy efficiency is achieved at the expense of very little loss in LL throughput as seen in Fig. 6.

\section{Conclusions}

We considered LL backoff algorithms along with LL ARQ for large round-trip delays on point-to-point wireless fading links. We proposed a GBN protocol with backoff, and presented a renewal-reward analysis of the throughput and energy efficiency of the proposed scheme. We showed that GBN with linear backoff results in energy savings of about $2 \mathrm{~dB}$ compared to GBN with no backoff. We also proposed and analyzed an adaptive GBN/SAW ARQ with backoff. The adaptive GBN/SAW with linear backoff achieves energy efficiency quite close to that of an ideal backoff scheme which

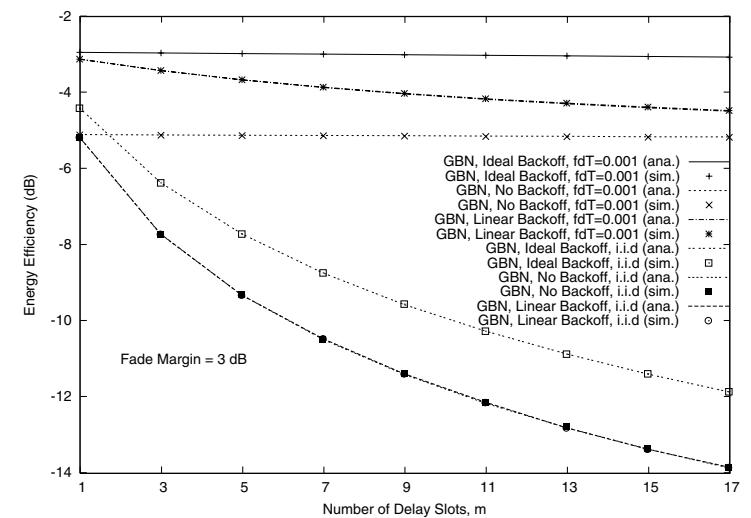

Fig. 4. Energy Efficiency as a function of number of round-trip delay slots, $m$, for GBN protocol without and with LL backoff.

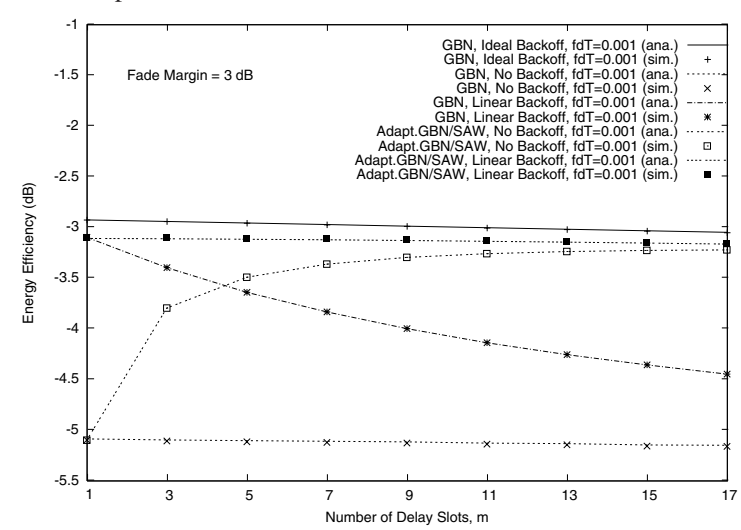

Fig. 5. LL Throughput vs number of round-trip delay slots, $m$, for adaptive GBN/SAW protocol and GBN protocol without and with LL backoff.

assumes a priori knowledge of the channel status in each slot.

\section{REFERENCES}

[1] W. C. Jakes, Jr., Microwave Mobile Communications, New York: JohnWiley \& Sons, 1974.

[2] P. M. Soni and A. Chockalingam, "Analysis of link-layer backoff schemes on point-to-point Markov fading links," vol. 51, no. 1, pp. 29-32, January 2003.

[3] P. M. Soni and A. Chockalingam, "Performance analysis of UDP with energy efficient link layer on Markov fading channels," IEEE Trans. Wireless Commun., vol. 1, no. 4, pp. 769-780, October 2002.

[4] M. Zorzi, R. R. Rao, and L. B. Milstein, "On the accuracy of a firstorder Markov model for data transmission on fading channels," Proc. IEEE ICUPC'95, Tokyo, November 1995.

[5] M. Zorzi and R. R. Rao, "Error control and energy consumption in communications for nomadic computing," IEEE Trans. Computers, vol. 46 , no. 3, pp. 279-289, March 1997.

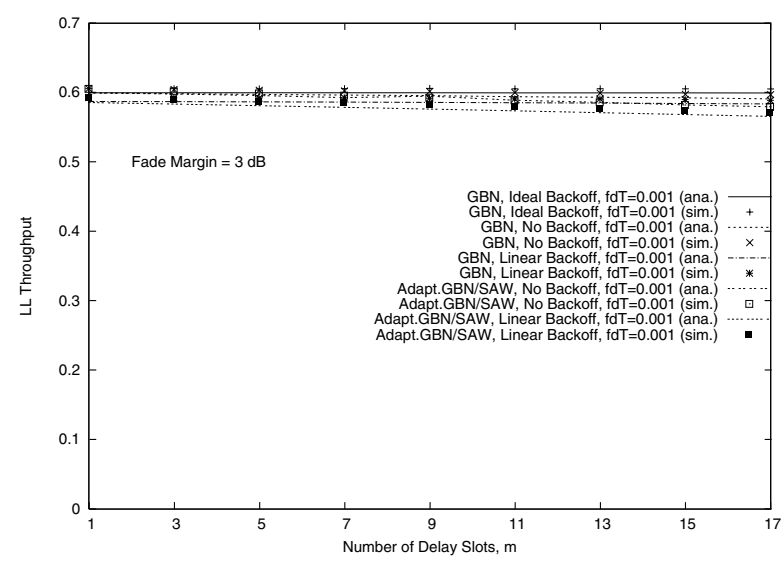

Fig. 6. Energy Efficiency vs number of round-trip delay slots, $m$, for adaptive GBN/SAW protocol and GBN protocol without and with LL backoff. 